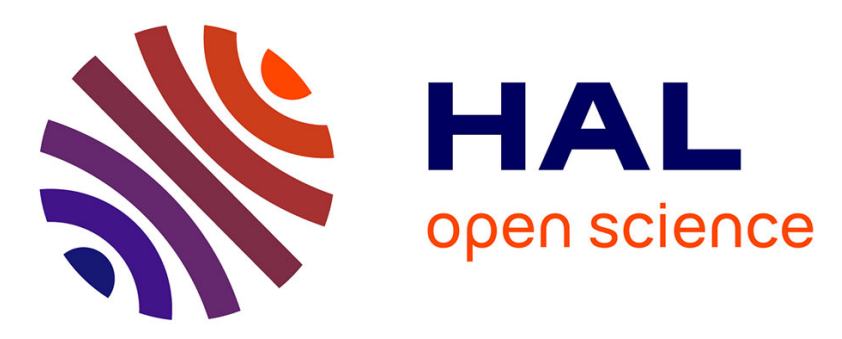

\title{
Prediction of the piezo-resistive behavior of rubber/carbon nanotube nanocomposites under quasi-static compression
}

Aravind Rajan Ayagara, André Langlet, Michel Gratton, Nourredine Aït Hocine

\section{To cite this version:}

Aravind Rajan Ayagara, André Langlet, Michel Gratton, Nourredine Aït Hocine. Prediction of the piezo-resistive behavior of rubber/carbon nanotube nanocomposites under quasistatic compression. Composites Part A: Applied Science and Manufacturing, In press, 10.1016/j.compositesa.2021.106428 . hal-03225397

\section{HAL Id: hal-03225397 \\ https://hal.science/hal-03225397}

Submitted on 12 May 2021

HAL is a multi-disciplinary open access archive for the deposit and dissemination of scientific research documents, whether they are published or not. The documents may come from teaching and research institutions in France or abroad, or from public or private research centers.
L'archive ouverte pluridisciplinaire HAL, est destinée au dépôt et à la diffusion de documents scientifiques de niveau recherche, publiés ou non, émanant des établissements d'enseignement et de recherche français ou étrangers, des laboratoires publics ou privés. 


\section{Journal Pre-proofs}

Prediction of the piezo-resistive behavior of rubber/carbon nanotube nanocomposites under quasi-static compression

Aravind Rajan AYAGARA, André LANGLET, Michel GRATTON, Nourredine AIT HOCINE

PII: S1359-835X(21)00151-2

DOI: https://doi.org/10.1016/j.compositesa.2021.106428

Reference: JCOMA 106428

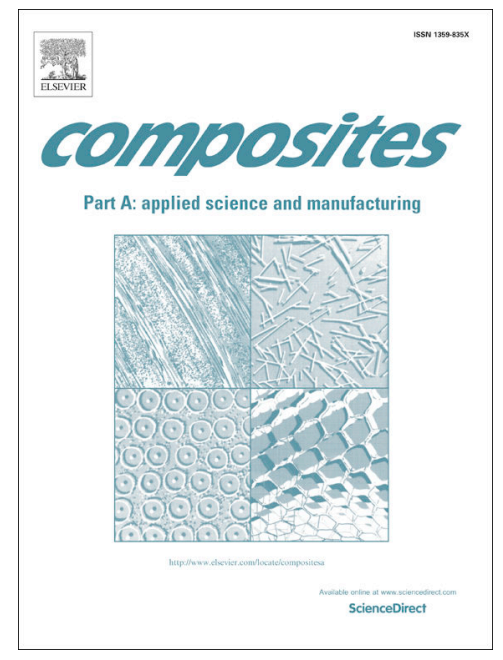

To appear in:

Composites: Part A

Received Date:

1 December 2020

Revised Date:

6 April 2021

Accepted Date:

12 April 2021

Please cite this article as: AYAGARA, A.R., LANGLET, A., GRATTON, M., HOCINE, N.A., Prediction of the piezo-resistive behavior of rubber/carbon nanotube nanocomposites under quasi-static compression, Composites: Part A (2021), doi: https://doi.org/10.1016/j.compositesa.2021.106428

This is a PDF file of an article that has undergone enhancements after acceptance, such as the addition of a cover page and metadata, and formatting for readability, but it is not yet the definitive version of record. This version will undergo additional copyediting, typesetting and review before it is published in its final form, but we are providing this version to give early visibility of the article. Please note that, during the production process, errors may be discovered which could affect the content, and all legal disclaimers that apply to the journal pertain.

(C) 2021 Published by Elsevier Ltd. 


\title{
Prediction of the piezo-resistive behavior of rubber/carbon nanotube nanocomposites under quasi-static compression*
}

\author{
Aravind Rajan AYAGARA ${ }^{\mathrm{a}, \mathrm{b}}$, André LANGLET ${ }^{\mathrm{a}, *}$, Michel GRATTON ${ }^{\mathrm{b}}$, Nourredine AIT \\ HOCINE $^{b}$ \\ ${ }^{a}$ Univ. Orléans, Univ. Tours, INSA CVL, LaMé, 63 av de lattre de Tassigny, 18020 Bourges-France \\ ${ }^{b}$ INSA CVL, Univ. Tours, Univ. Orléans, LaMé, 3 rue de la Chocolaterie, CS 23410, 41034 Blois Cedex, \\ France
}

\begin{abstract}
This paper presents the prediction of piezo-resistive behavior under quasi-static uniaxial compression loads using the Percolation Excluded Volume (PEV) approach. The PEV approach is supplemented by Finite Element (FE) simulations to calculate the displacements of Carbon Nano-Tubes (CNTs) embedded in a polymer matrix. The number of contacts between CNTs is determined by the minimum distance threshold (tunnelling distance). The FE model is based on experimental parameters and CNT data. The simulations carried out using the LS-DYNA solver made it possible to calculate the variation of electrical resistance, which was found to be in accordance with its experimental counterpart. The resistance increases when the CNTs are mostly oriented orthogonally to the loading direction. For other orientations, the resistance decreases.
\end{abstract}

Keywords: A. Nanocomposites, A. Polymer-Matrix Composites, B. Electrical properties, C.Numerical analysis

${ }^{\star}$ Draft Manuscript, please do not cite without authors permissions

${ }^{*}$ Corresponding author

Email address: andre.langlet@univ-orleans.fr (André LANGLET) 


\section{List of Tables}

1 Input parameters of CNT for $h\left(\right.$ Eq. 12) and $\sigma_{\mu}$ (Eq. 10) . . . . . . . . . . 20

\section{List of Figures}

1 Concept of effective CNT adapted from $[24] \ldots \ldots \ldots$

2 Samples and experimental set-up used $[39] \ldots \ldots \ldots \ldots$

3 Comparison of numerical stress strain curve obtained from *MAT-181 LS-DYNA 12

4 Schematic of a single CNT in a cubic VE . . . . . . . . . . . . . . 13

5 Contact due to proximity of CNTs below $d_{\text {tun }} \ldots \ldots \ldots \ldots$

6 Changes in the CNT coordinates . . . . . . . . . . . . . 17

7 Effect of $\theta_{0}$ on $h$ in 10 different micro-structures . . . . . . . . . . . . . 18

8 Comparison of number of contacts from three different methods . . . . . . . . 19

9 Experimental percolation threshold from [39] . . . . . . . . . . . . . . . . . . . 19

10 Contacts, conductivity and percolation threshold predicted by PEV approach . 21

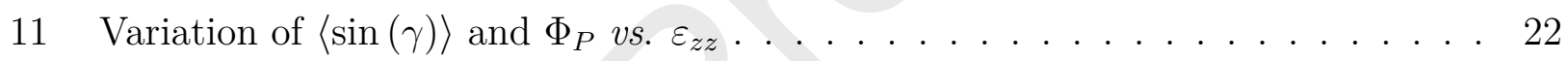

12 Variation of number of contacts with strain for different angular parameters $\theta_{\max }$

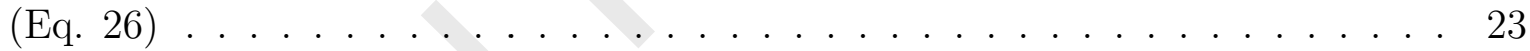

13 Evolution of conductivity and resistivity with mechanical strain . . . . . . . . 24

14 Variation of $\Delta R$ at the macro-scale with respect to strain and its comparison with experimental results . . . . . . . . . . . . . . . . 25 
List of figure files

Figure 1

Figure 2

Figure 3

Figure 4

Figure 5

Figure 6

Figure 7

Figure 8

Figure 9

Figure 10

Figure 11

Figure 12

Figure 13

Figure 14 (a): A_schematic_of_wavy_and_straight_CNT.eps

(b): Effective_CNT_CNT_with_interphase.eps

(a): Overmolded_EPDM_CB_MWCNT_sample.eps

(b): Compressive_test_set_up.eps

Stress_Strain_Curve_Expt_Dyna.eps

Schematic_of_CNT_in_a_cubic_VE.eps

Contact_due_to_proximity_of_CNTs_below_dtun.eps

Changes_in_the_CNT_coordinates_R1.eps

Effect_of_theta0_on_h_in_10_different_microstructures.eps Comparison_of_number_of_contacts_from_three_models.eps Experimental_percolation_threshold_from_Penvern2020.eps

(a): Number_of_contacts_vs_phiCNT.eps

(b): Initial_conductivity_vs_phiCNT.eps

(a): Variation_of_sin_gamma_vs_strain.eps

(b): Variation_of_PhiP_vs_strain.eps

(a): Effect_of_CNT_orientation_on_m_vs_strain_REV1.eps

(b): m_for_thetamax_0degree_and_polynomial_fit.eps

(a): Conductivity_vs_strain.eps

(b): Resistivity_vs_strain.eps

(a): Effect_of_Orientation_on_Delta_R_REV1.eps

(b): Delta_R_for_thetamax_Odegree.eps 


\section{Introduction}

The incorporation of Nanomaterials, for example CNTs or Graphene, in a matrix creates a composite whose properties may differ significantly from that of the matrix alone [1]. The interesting capacity to convert thermal to electric energy in conductive material (Seebeck effect) may be exploited in carbon-material-based organic thermoelectric composites (review [2]). The influences of the physico-chemical phenomenon at the interface is crucial in the enhancement of the strength, the thermal conductivity and stability of the composites, as shown by the results of [3], [4], and [5]. A stochastic multiscale method to quantify the parameters influencing the mechanical properties (Young Modulus, Poisson's ratio) of polymer nanocomposites (PNCs) was proposed by [6].

As CNTs are electrically conductive, PNCs also exhibit interesting features for flexible sensor designs (see for example [7]) even in the large strain domain. It is one of the reason why Polymer nanocomposites (PNCs) have attracted considerable attention in recent years, especially those including CNTs. The present study concerns the prediction of the piezoresistive response of PNCs under compressive loads.

PNCs are categorized into two categories namely (i) Single Filler Polymer Nano-Composites (SFPNC) and Multiple Filler Polymer Nano-Composites (MFPNC) or Hybrid Polymer NanoComposites (HPNC).

Among the filler materials, carbon-based fillers are the most frequently used owing to their manufacturing cost to efficiency ratio or processing cost to efficiency ratio. According to the literature, four types of carbon fillers are often used: (i) Graphite (G), (ii) Carbon Black (CB), (iii) Carbon Fiber (CF) and (iv) Carbon-Nano Tubes (CNTs) [8]. Other types of carbon-based fillers include Graphene sheets, Nanowires etc., [9].

The fillers can be classified in two categories: (i) particulate fillers i.e. particles with spherical or ellipsoid geometrical shapes and (ii) fibrous fillers i.e. fillers with a large aspect ratio such as layers of curved cylinders. For example, fillers such as $G$ or silica fall in the first category whereas fibers such as $\mathrm{CF}$ or CNT fall into the latter category. As CNTs are long, they act as long-distance charge carriers, thereby contributing to conductivity across the volume as opposed to $\mathrm{G}$ filler that contribute to local conductivity $[10,11,8]$ and $[12]$.

Several authors have proposed methods to estimate the conductivity of PNCs. One of 
the most common methods that can be applied to SFPNCs is the Percolation theory. The name itself suggests that such a model is based on the estimation of the percolation threshold. Quoting [13]: the percolation threshold is a critical probability at which a connected network of sites is formed which spans the sample. Here, the notion of sites represents conducting particles. To estimate the percolation threshold of PNCs, filler particles are generated in a volume either randomly or following a user-specified distribution, then the system of particles is checked for connectivity between two particles. A cluster is defined when any two of these particles come into contact. The quantity of filler particles at which at least one cluster spans the entire sample gives us an estimate of the percolation threshold.

Since the connectivity between conductive particles is influenced by their spatial distribution in the polymer matrix, the argument of excluded volume has been introduced in the theory of percolation. Excluded volume is the volume around an object which a similar object cannot enter unless the interpenetration of two objects is permitted. Extensive research can be found regarding the excluded volume of different geometrical shapes $[14,15,16]$ and [17]. The base of the excluded volume was first proposed by [15], and consisted of an elliptic integral, that can be adapted to various situations leading to case specific formulae, as used in this study. The percolation threshold is then dependent on the geometry of the filler material. The relation between the percolation threshold and geometry was first proposed by [18]. This relation was further extended by [14] stating that the number of particles per unit volume at the percolation threshold $q_{p}$ is inversely proportional to the excluded volume $V_{e x}$.

The proportionality $q_{p} \propto 1 / V_{e x}$ is elucidated by replacing it with the volume of the filler particle. Three dimensional fillers with a high aspect ratio such as fibers or CNTs are represented as capped cylinders also known as spherocylinders. A spherocylinder is a cylinder enclosed by a hemisphere of the same radius at each extremity. The use of capped cylinders was justified by [19] for a more realistic representation of CNTs. The excluded volume is not only a geometrical parameter, rather it plays a crucial role in the prediction of the percolation threshold. Studies regarding the influence of filler geometry are [20] and [21] in the case of two-dimensional systems and [16, 19, 22, 23] in case of three-dimensional (3D) systems.

The dependency of the percolation threshold of sticks or cylinders on their aspect ratio and anisotropy of the stick orientation in the polymer matrix highlighted by [19] was taken 
a step further by [16]. They proposed a model to predict the percolation threshold of high aspect ratio fiber systems. Two important assumptions were made in their work: (i) the fibers do not come into contact physically, and (ii) fibers cannot interpenetrate. These assumptions were taken into account through CNT models that they refer to as Soft core and Hard core cylinder models. Even though the thickness of interphase regions around a CNT was considered in the form of a shell, the waviness of a CNT embedded in a polymer matrix was not taken into account. Rather, their work laid the foundation for considering the geometrical effects of a CNT/ fiber on the excluded volume and therefore on the percolation threshold. These geometrical properties (interphase thickness and waviness of high aspect ratio fibers / CNTs) were considered by [24] in the form of the effective CNT model. The formula for the excluded volume of hard-core cylinders proposed by [16] was modified by [24] to an effective CNT.

The electrical behavior of PNCs is mainly due to electron hopping between the conductive fillers when they are at a distance known as the tunneling distance. As per [25], the tunneling distance $d_{t u n}$ is $1.4 \mathrm{~nm}$, whereas [26] presented an alternative method to calculate the $d_{t u n}$. Apart from these studies, [27, 28, 29, 30, 31] and [32] considered the tunneling resistance to calculate an equivalent resistance of the nanocomposite.

The other important aspect for modelling the conductivity of PNCs is the number of contacts between the conductive nanoparticles present in a given volume. The tunnelling distance $d_{\text {tun }}$ plays a crucial role in determining the number of contacts between filler particles. The relationship between electrical resistivity or electrical conductivity and the number of contacts dates back to 1997 [13]. An equation relating the number of contacts to the excluded volume, the filler volume fraction and filler excluded volume, was proposed by [29]. One of the recent articles that considers the number of contacts alongside the interphase regions and waviness to predict the electrical conductivity is [24].

The aforementioned studies do not consider the modification of spatial distributions of the CNTs when external mechanical loads are applied to the PNC. Two methods are commonly used to study the related changes in electrical conductivity, or "piezo-resistive behavior" of PNCs. The first one is the Fiber Reorientation Model (FRM) as published by [23]. Applications and improvements of the FRM can be found in [33] and [32]. The limitations of FRM can be overcome by micro mechanical models. Recent studies concerning FE based micro-mechanical 
models are $[34,35,36,37]$ and [38]. Yet the extension of these micro-mechanical models to the macro-scale becomes prohibitive in terms of numerical resources.

In this study, we derive a model based on the PEV approach previously presented by [24] and related studies (mainly [16], [13], [29]). The present model enable takes into account the effect of the field displacements and strains caused by compressive static loads. Mechanical fields are computed by FE simulations and are processed to approximate the displacement of CNTs modelled as rigid 3D cylinders. The following parameters are updated for the computed stress-strain states: variation of number of contacts, change in the CNTs orientation of a micro-structure and therefore its change in excluded volume. By doing so, we can propose a prediction for the evolution of electrical conductivity with respect to mechanical strain. The predicted numerical results are compared to their experimental counterpart.

\section{Percolation excluded volume approach: theoretical formulation}

Based on the hypotheses presented by [14], [16] put forth the relation between percolation threshold and excluded volume for soft core cylinders and hard core cylinders. This important assumption, still in use today, gives a relation between the percolation threshold $\left(\Phi_{P}\right)$ and the average excluded volume $\left(V_{e x}\right)$ :

$$
\Phi_{P}=\frac{V_{C N T}}{V_{e x}}
$$

The volume $V_{C N T}$ in Eq. 1 is the sum of the volume of a carbon nanotube cylinder and twice the volume of a hemisphere, expressed as:

$$
V_{C N T}=\pi r^{2} \ell+2 \times \frac{2}{3} \pi r^{3}
$$

where $\ell$ and $r$ are CNT length and radius respectively. The excluded volume of a system of spherocylinders is expressed as follows:

$$
V_{e x}=\frac{32}{3} \pi r^{3}\left[1+\frac{3}{4}\left(\frac{\ell}{r}\right)+\frac{3}{8 \pi}\langle\sin (\gamma)\rangle\left(\frac{\ell}{r}\right)^{2}\right]
$$

Eq. 3 indicates that $V_{e x}$ is dependent on the relative angle $\gamma$ between any given pair of CNTs. The parameter $\langle\sin (\gamma)\rangle$ is the mean of $\sin (\gamma)$ among all pairs of CNTs. As per [16] and [24] for randomly distributed CNTs, $\langle\sin (\gamma)\rangle \approx \pi / 4$. 
It is well established that the high surface area of CNTs forms an intermediate zone with the surrounding polymer known as interphase and also tends to entangle when induced in a polymer matrix, thus influencing the geometry of CNTs. The new equivalent dimensions $\left(\ell_{e q}\right.$ and $\left.r_{e q}\right)$ are expressed as:

$$
\begin{aligned}
& \ell_{e q}=\frac{\ell}{s} \\
& r_{e q}=r+h
\end{aligned}
$$

where $\ell$ and $r$ are original length and original radius of CNT, $s$ is the waviness parameter and $h$ is the interphase thickness. The waviness has an effect on the length of the CNT as shown in Fig. 1a whereas the interphase influences the effective radius or cross sectional area as shown in Fig. 1b. These aspects of CNTs were first considered by [24] through their concept of effective CNT.

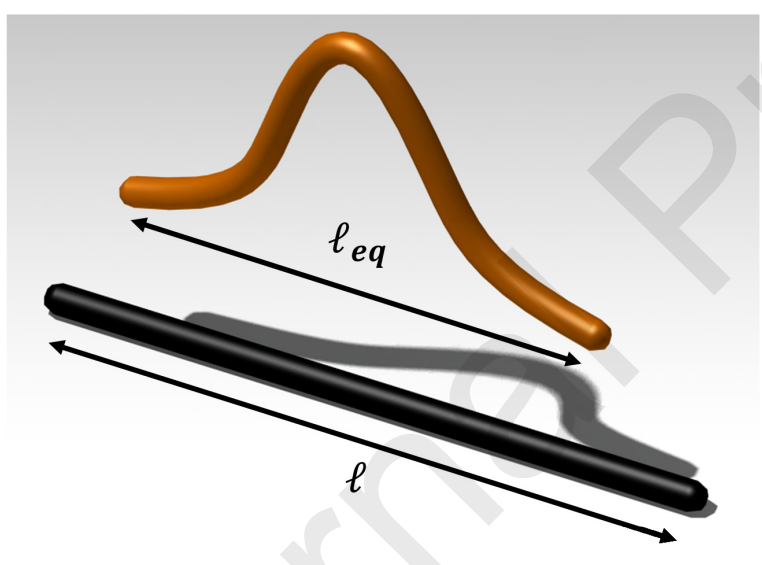

(a) A Schematic of wavy and straight CNT

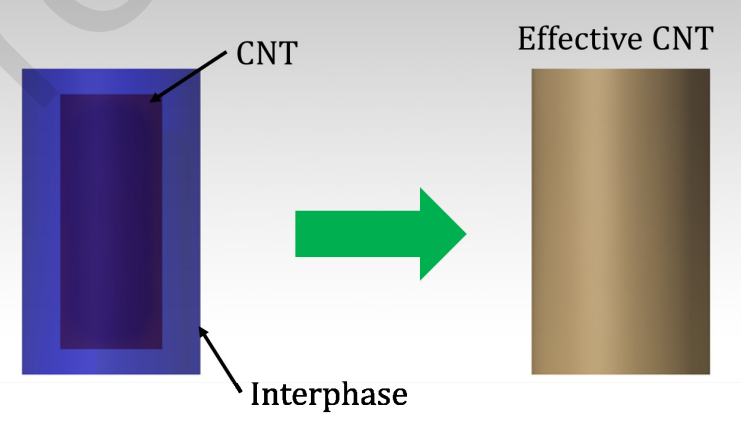

(b) Effective CNT with interface

Figure 1: Concept of effective CNT adapted from [24]

For any given distribution of CNTs, the excluded volume for an effective CNT is expressed as:

$$
V_{e x}=\frac{32}{3} \pi r_{e q}^{3}\left[1+\frac{3}{4}\left(\frac{\ell_{e q}}{r_{e q}}\right)+\frac{3}{8 \pi}\langle\sin (\gamma)\rangle\left(\frac{\ell_{e q}}{r_{e q}}\right)^{2}\right]
$$

The volume fraction effective $\operatorname{CNT}\left(\varphi_{\text {eff }}\right)$ is a part of the volume fraction $\varphi_{C N T}$, which is 
the ratio of total CNT volume to total volume of the sample.

$$
\varphi_{e f f}=\frac{(r+h)^{2}(\ell / s+2 h)}{r^{2} \ell / s} \times \varphi_{C N T}
$$

If the CNTs are assumed to be straight and no interphase is considered, then $\varphi_{\text {eff }}=\varphi_{C N T}$. The next step according to [24] is to find the fraction $f$ of CNTs that contribute to forming an effective conductive path. The parameter $f$ is a function of $\varphi_{\text {eff }}$ and $\Phi_{P}$

$$
f=\frac{\varphi_{e f f}^{1 / 3}-\Phi_{P}^{1 / 3}}{1-\Phi_{P}^{1 / 3}}
$$

$f$ varies between 0 and 1 .

Another important parameter that has an influence on conductivity is the number of contacts $m$, which is linked to the conductivity $\sigma$ through a parameter $\chi$. The expression for $\chi$ was first introduced by [13] as follows:

$$
\chi=\frac{1}{0.59+0.15 m}
$$

Thanks to this expression, [24] proposed a relation to estimate the electrical conductivity for an effective CNT:

$$
\sigma=\frac{f \varphi_{e f f} d_{c} \ell \sigma_{C N T}}{3 \pi r^{2} \chi s}
$$

where $d_{c}$ is the diameter of contact and $\sigma_{C N T}$ is the intrinsic electrical conductivity of a CNT. Eq. 10 shows that the electrical conductivity is influenced by $f$ and $\chi$. Thus, it is directly reliant on the geometry through $\ell, r$ and $s$. Simultaneously, it is indirectly reliant on the percolation threshold $\Phi_{P}$ and number of contacts $m$.

The interphase thickness can be determined by rearranging Eq. 1 as follows:

$$
V_{e x}-\frac{V_{C N T}}{\Phi_{P}}=0
$$

Replacing $V_{e x}$ in Eq. 11 with that in Eq. 6 and substituting the expressions of $\ell_{e q}$ and $r_{e q}$ (Eq. 4 and Eq. 5 respectively), leads to :

$$
\frac{32}{3} \pi(r+h)^{3}\left[1+\frac{3}{4}\left(\frac{\ell / s}{r+h}\right)+\frac{3}{8 \pi}\langle\sin (\gamma)\rangle\left(\frac{\ell / s}{r+h}\right)^{2}\right]-\frac{V_{C N T}}{\Phi_{P}}=0
$$


If $\ell, r, s,\langle\sin (\gamma)\rangle$ and $\Phi_{P}$ are known then Eq. 12 is a third degree polynomial in terms of $(r+h)$, allowing the estimation of the interphase thickness $h$.

\section{Experimental method}

The experimental study was carried out on an EPDM matrix reinforced with CB particles obtained from fast extraction furnace process and multi-wall CNTs produced using chemical vapor deposition. All samples consisted of $17.4 \%$ volume for $\mathrm{CB}$ (which is well above the percolation threshold of $\mathrm{CB}$ composites) and $0 \%$ vol, $1 \%$ vol, $3 \%$ vol and $5 \%$ vol of CNT respectively. For the ease of electrical measurement, two $3 \mathrm{~mm}$ thick cylindrical brass electrodes were over-molded on the top and bottom of the sample as shown in Fig. 2a.

The total length of the sample was $16 \mathrm{~mm}$, and its diameter was $20 \mathrm{~mm}$. The effective length of the composites was $10 \mathrm{~mm}$, which shall be used in hereafter.

Electrical impedance $Z$ was measured through the brass electrodes. An electrical current $I$ in the range of $10 \mu \mathrm{A}$ to $3 \mathrm{~mA}$ was input through a linear measurement device. Thanks to this electronic scheme (see [39]) the range of measurement can be adapted by choosing an appropriate input current value. This setup gives the electrical voltage $V$ as output, which in turn is used to calculate the electrical impedance at the macro-scale $Z=V / I$.

Monotonic uni-axial compression tests were carried out on a computer controlled MTS tension/compression machine equipped with a load cell of $10 \mathrm{kN}$. The TWE Elite software was used to control the machine. A schematic of the test setup is presented in Fig. 2b. The samples were subjected to quasi-static uni-axial compression by displacement of the cross-head. The samples were placed in a notch with an insulating adhesive. The upper cross-head was connected to a two-part alignment device separated by a thin film of grease. This alignment device ensured that the sample was always subjected to pure uni-axial compression. 


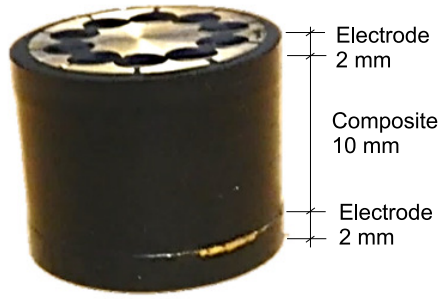

(a) Overmolded EPDM/CB/CNT composite

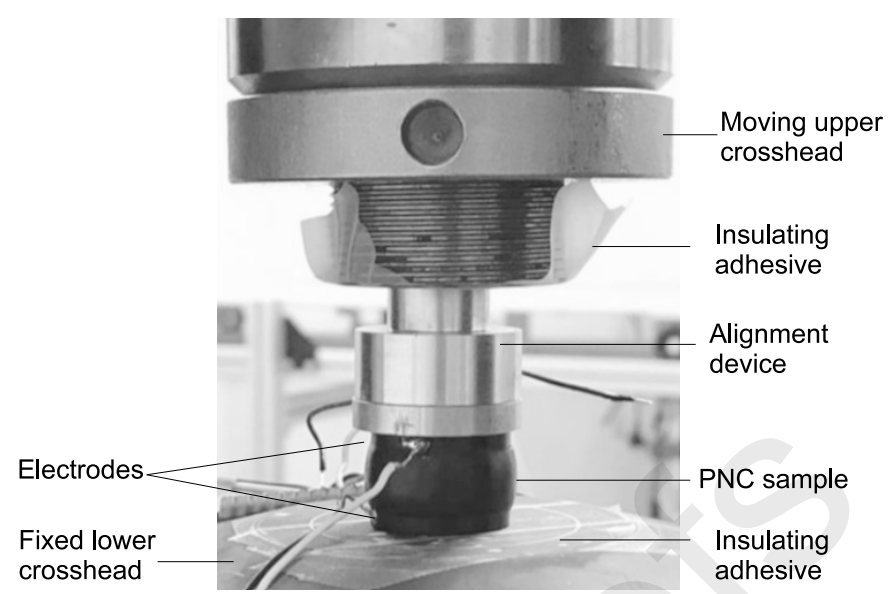

(b) Photograph of the quasi-static compression test bench

Figure 2: Samples and experimental set-up used [39]

\section{Finite Element Simulations}

\subsection{Constitutive Law}

The Finite Element (FE) simulations were executed using the implicit general solver of the LS Dyna mechanical solver on a sample of radius $r_{S}=10 \mathrm{~mm}$ and length $\ell_{S}=10 \mathrm{~mm}$. The Ogden hyper-elastic constitutive model was used.

$$
\begin{aligned}
W= & \sum_{m=1}^{n} \frac{\mu_{m}}{\alpha_{m}}\left(\tilde{\lambda}_{1}^{\alpha_{m}}+\tilde{\lambda}_{2}^{\alpha_{m}}+\tilde{\lambda}_{3}^{\alpha_{m}}-3\right) \\
& +\frac{1}{2} K(J-1)^{2} \\
\tilde{\lambda}_{i}= & \frac{\lambda_{i}}{J^{1 / 3}} \\
J= & \lambda_{1} \lambda_{2} \lambda_{3}
\end{aligned}
$$

$\lambda_{1}, \lambda_{2}$ and $\lambda_{3}$ are isochoric principal stretches. The material parameters $\mu_{m}$ and $\alpha_{m}$ are found by least square fitting of the user input experimental stress as a function of strain. The material bulk modulus and relative volume change are represented by $K$ and $J$.

The experimental engineering stress-strain curve $\sigma(\varepsilon)$ ( $\varepsilon$ is the change in length to original length ratio) was provided by [39]. This enables to determine the material constants $\mu_{m}$ and 
$\alpha_{m}$. The Cauchy stress components $\sigma_{i j}$ are then calculated using the following equation:

$$
\sigma_{i j}=J^{-1} q_{i k} q_{j l} \lambda_{i} \frac{\partial W}{\partial \lambda_{i}}
$$

where $q$ is the orthogonal tensor containing the eigenvectors of the principal basis. In order to verify that the constitutive law is correctly implemented, a single element was subjected to compression and the numerical stress strain response was compared to the experimental input curve (Fig. 3). The numerical results fit the experimental curve well, confirming a correct implementation of the constitutive law.

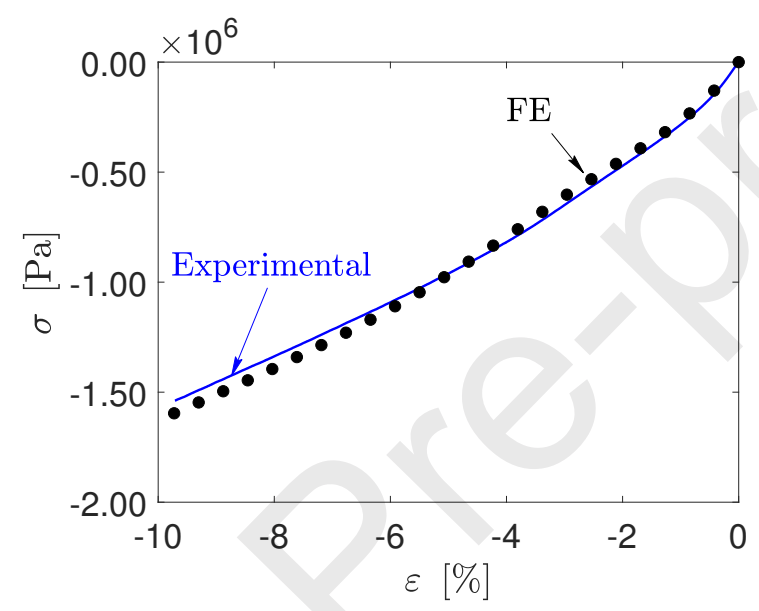

Figure 3: Comparison of numerical stress strain curve obtained from *MAT-181 LS-DyNA

\subsection{Generation of micro-structure}

Here, we present the algorithm used to generate the micro-structure. The dimensions of CNT considered were: length $\ell=5 \mu m$ and diameter $d=50 \mathrm{~nm}$. A cubic volume element (VE) with a length 2.2 times the length of CNT is chosen based on the conclusions of [40] and [41]. An example of a cubic VE with randomly oriented CNTs is presented in Fig. 4

$$
L_{x}=L_{y}=L_{z}=2.2 \times \ell=11 \mu \mathrm{m}
$$




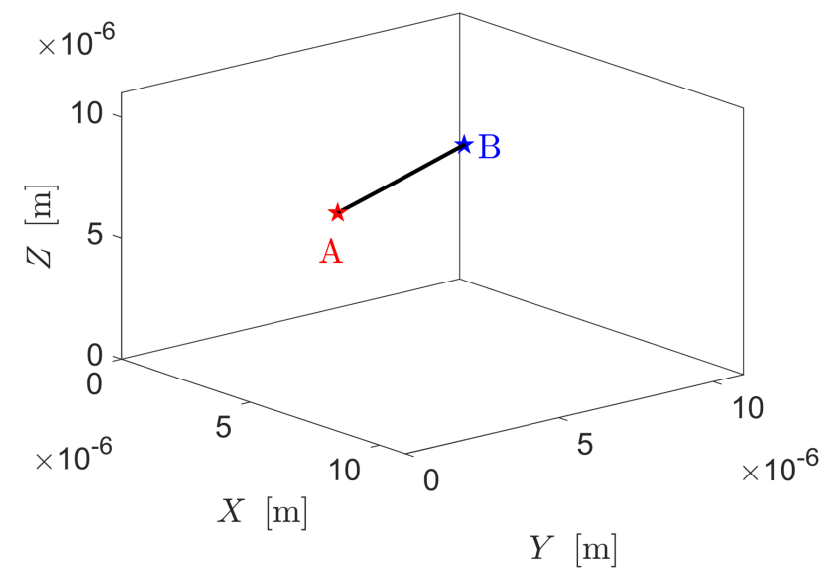

Figure 4: Schematic of a single CNT in a cubic VE

The dimensions of $\operatorname{VE}\left(L_{x}, L_{y}\right.$ and $\left.L_{z}\right)$, dimensions of $\operatorname{CNT}(\ell, d)$ and the volume fraction of $\mathrm{CNT}\left(\varphi_{C N T}\right)$ are required to generate a micro-structure.

Let us consider a CNT with extremities $A$ and $B$ in a Cartesian 3D space. Its position is well described through the coordinates of its extremities $A, B$ and its orientation is identified through the angle $\theta_{0}$ with respect to $X$ axis and the angle $\phi_{0}$ with respect to $Z$ axis. The following set of equations was used to generate each $\mathrm{CNT}$ in $3 \mathrm{D}$ space. The reoccurring parameter rand is a random number. The "rand" function used generates uniformly distributed pseudorandom numbers.

The expressions for $\theta_{0}$ and $\phi_{0}$ for a random distribution are from [33].

$$
\begin{aligned}
x_{0}^{A} & =L_{x} \times \text { rand } \\
y_{0}^{A} & =L_{y} \times \text { rand } \\
z_{0}^{A} & =L_{z} \times \text { rand } \\
\theta_{0} & =2 \pi \times \text { rand } \\
\phi_{0} & =\arccos (2 \times \text { rand }-1) \\
x_{0}^{B} & =x_{0}^{A}+\ell_{C N T} \sin \phi_{0} \cos \theta_{0} \\
y_{0}^{B} & =y_{0}^{A}+\ell_{C N T} \sin \theta_{0} \sin \phi_{0} \\
z_{0}^{B} & =z_{0}^{A}+\ell_{C N T} \cos \phi_{0}
\end{aligned}
$$


In order to have a user specified orientation and distribution of CNTs, it would be enough to change the expressions for angles $\theta_{0}$ and $\phi_{0}$ in Eq. 21 and Eq. 22. According to [40], this could be done by setting a parameter $\theta_{\max }$ with respect to $X$ axis. This leads to the following set of equations:

$$
\begin{aligned}
& \theta_{0}=\arccos \left(1-\cos \theta_{\max }\right) \times \text { rand }+\theta_{\max } \\
& \phi_{0}=2 \pi \times \text { rand }
\end{aligned}
$$

The relative angle $\gamma_{i j}$ between two CNTs (say $i$ and $j$ ) is calculated by the dot product of the CNT unit vectors $\mathbf{u}_{\mathbf{i}}$ and $\mathbf{u}_{\mathbf{j}}$ :

$$
\gamma_{i j}=\arccos \left(\mathbf{u}_{\mathbf{i}} \cdot \mathbf{u}_{\mathbf{j}}\right)
$$

\subsection{Contacts between a CNT pair}

The work of [24] is based on a Hard Core CNT model first proposed by [16]. The following hypotheses were considered :

- CNTs do not overlap (only one contact point is authorized)

- The CNTs are assumed not to interpenetrate whilst the interphase regions can interpenetrate.

[13] validated a model predicting conductivity using contacts. They tested different types of contacts i.e. end-to-end, end-to-body and body-to-body, but little information was provided on the overlapping and interpenetration of sticks. The maximum number of contacts was inferred as 15 .

More recently, [29] deduced a relation for contacts

$$
m=\left(\frac{\varphi_{C N T}}{2 V_{C N T}}\right) \times V_{e x}
$$

where $\varphi_{C N T}$ is the volume fraction, $V_{C N T}$ is volume of CNT and $V_{e x}$ is the excluded volume. But this relation is based on a soft core approach i.e. the CNTs can overlap.

In the present work, we propose a criterion to determine contacts based on the minimum distance between a CNT pair in the micro-structure. This can be done in two ways: (i) by 
considering the distance between two CNT axes and (ii) by considering the surface of the cylinder.

(i) Since the first approach takes only the CNT axis into account, it would suffice to represent the CNTs as sticks. This approach is relatively easy to implement. However, it lacks the precision to consider the curvature of the CNT surface. This approach is referred to as Sticks

(ii) The second approach considers solid cylinders and thus the surface and the edges of the cylinders. This requires a specialized algorithm for detecting the nearest points between two cylinders in three dimensions. This is done with minor modifications to the MATLAB function proposed by [42]. This approach is referred to as Cylinders.

Interpenetration of CNTs was forbidden. However, the interphase regions may interpenetrate. Interpenetration is detected by a negative distance.

Expressed in terms of distance, let us assume that $d_{i j}$ is the distance between two CNTs $i$ and $j$. Then, a contact is assumed to exist only if:

$$
0<d_{i j} \leq d_{\text {tun }}
$$

Therefore, a tunneling contact is purely due to the proximity of CNTs, leading to a distance less than the tunneling distance, as illustrated in Fig. 5.

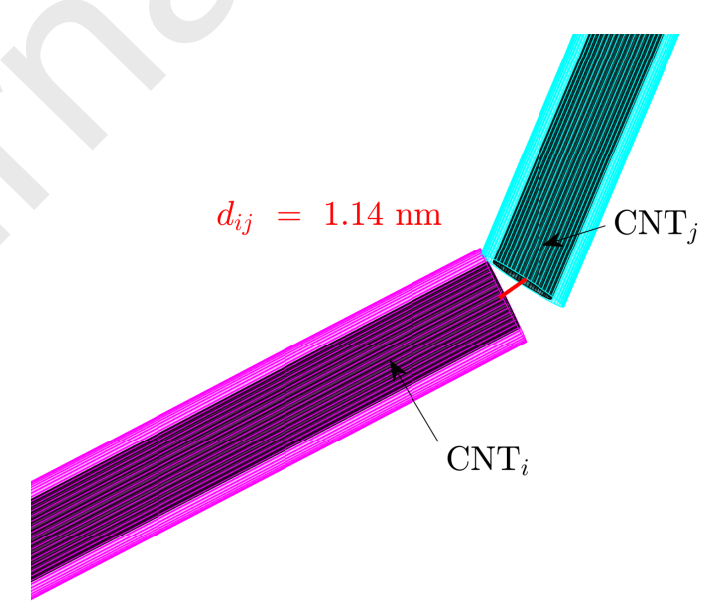

Figure 5: Contact due to proximity of CNTs below $d_{t u n}$ 


\subsection{Evaluation of CNT displacement field}

The cubic VE is meshed with an 8 node solid brick element. The nodes at $Z=11[\mu \mathrm{m}]$ are subjected to a monotonic increasing displacement $\left(0.0 \mathrm{~m}\right.$ at $0 \%$ strain increasing to $1 \times 10^{-6} \mathrm{~m}$ at $10 \%$ strain). The nodes at $Z=0[\mu \mathrm{m}]$ have a $Z$ displacement constrained to 0 .

The nodal displacements of the mesh are used to estimate the CNT displacements at each stress state (numbered $k$ ). The following procedure was chosen to evaluate the displacement of each CNT. At the $k-1$ stress state, we find within which mesh elements the centre point $C^{k-1}$, the extremities $A^{k-1}$ and $B^{k-1}$, of each CNT, are located. Then at the following state, $k$, the new coordinates of points $a^{k}, C^{k}$, and $b^{k}$, are interpolated from the known node displacements of the elements. The interpolation functions are classical FE interpolation functions. Knowing the temporary points $a^{k}$ and $b^{k}$, the unit vector $\mathbf{u}^{k}$ is determined:

$$
\mathbf{u}^{k}=\frac{b^{k}-a^{k}}{\left\|b^{k}-a^{k}\right\|}
$$

The positions of CNT extremities are updated so that the CNT length constantly remains equal to $\ell_{C N T}$. This is done by calculating:

$$
\begin{aligned}
A^{k} & =C^{k}-\frac{\ell}{2} \mathbf{u}^{k} \\
B^{k} & =C^{k}+\frac{\ell}{2} \mathbf{u}^{k}
\end{aligned}
$$

Note that points $A^{k}, C^{k}$, and $B^{k}$ are necessarily aligned although $a^{k}, C^{k}$ and $b^{k}$ may not be aligned. This procedure is illustrated in Fig. 6. 


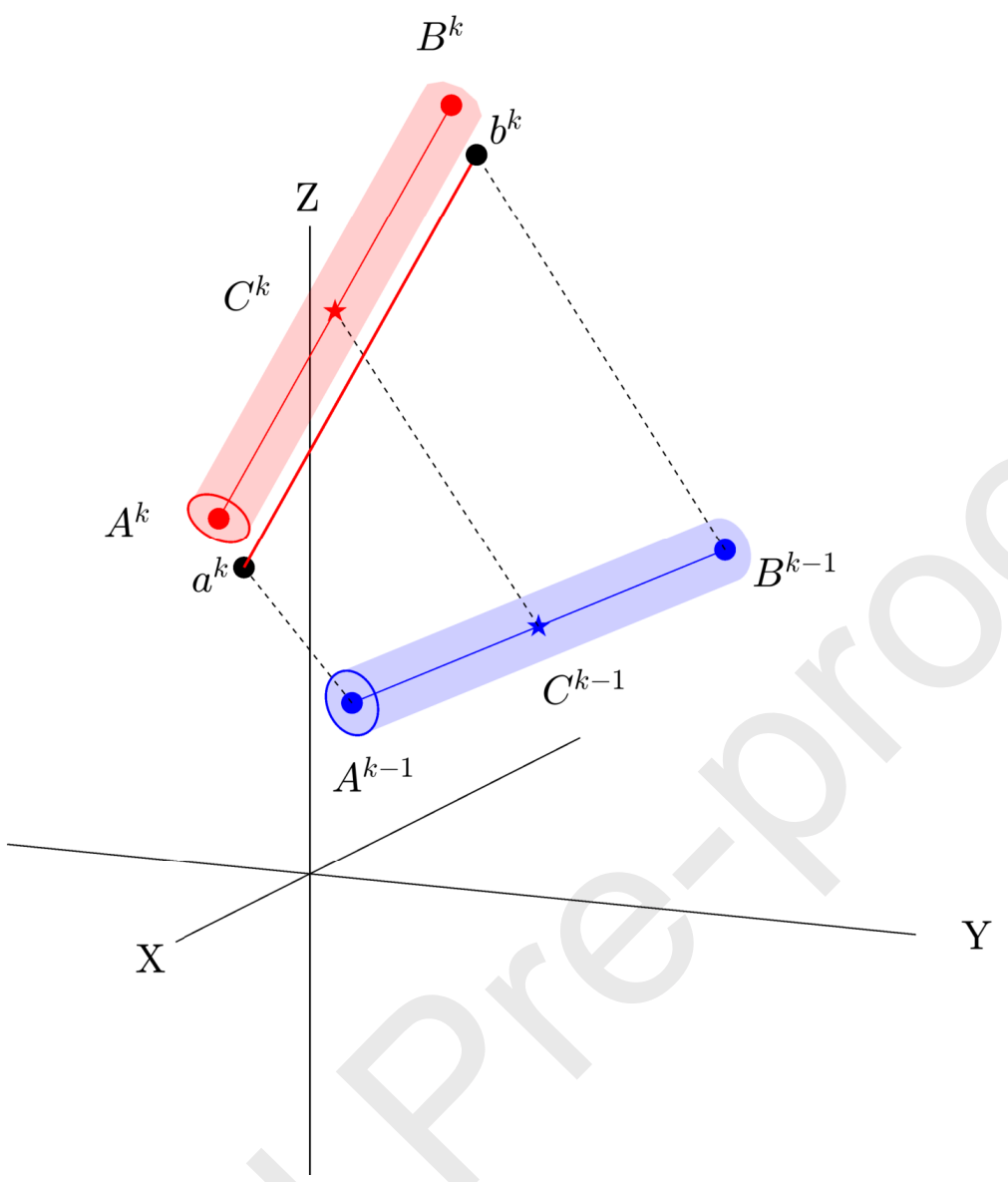

Figure 6: Changes in the CNT coordinates

This change in extremity coordinates will in fact induce a change in the CNT vector and thus the relative angle $\gamma_{i j}^{k}$. Since $\gamma_{i j}^{k}$ is different from its initial value $\gamma_{i j}^{k-1}$, we will have a different value of $V_{e x}^{k}$ (according to Eq. 6). This may change the percolation threshold $\Phi_{P}^{k}$. Similar to these parameters, the displacement field of CNTs does influences the distances and therefore the number of contacts $m$. Thus, the distances and contacts are updated for every stress state. 


\section{Results and Discussion}

\subsection{PEV approach results without stress (initial state)}

\subsubsection{Effect of CNT orientation}

The orientation of CNTs can be changed through Eq. 26 and Eq. 27. A different value of $\theta_{0}$, will give a different value of $\langle\sin (\gamma)\rangle$. Since the interphase $h$ is determined using Eq. 12, different values of $\langle\sin (\gamma)\rangle$ for different $\theta_{0}$ will lead to a different $h$. Fig. 7 , presents the variation of $h$ vs. $\theta_{0}$.

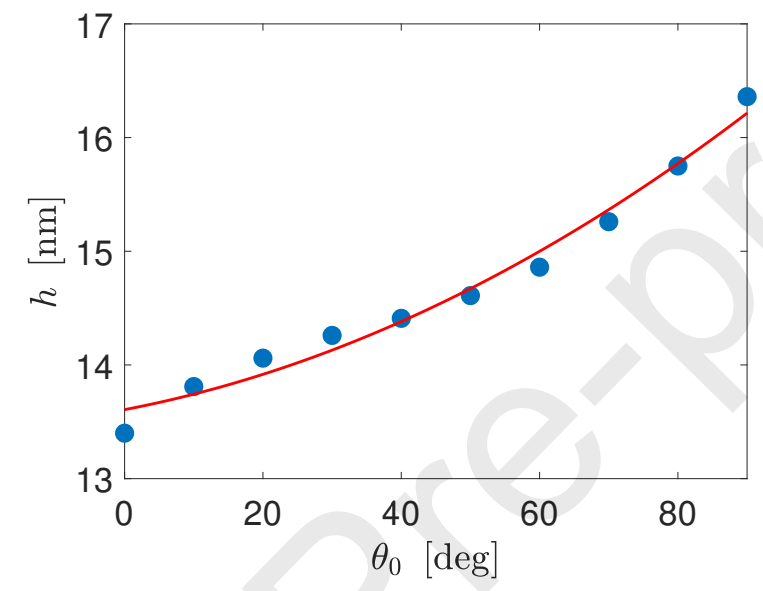

Figure 7: Effect of $\theta_{0}$ on $h$ in 10 different micro-structures

As the angle $\theta_{0}$ increases, the parameter $\langle\sin (\gamma)\rangle$ decreases. In order to compensate for this change in angle and to have a percolation threshold of $1.8 \%$, the interphase thickness increases. The lowest interphase value is obtained for a randomly distributed micro-structure $h=10.36 \mathrm{~nm}$ which has the highest $\langle\sin (\gamma)\rangle=0.7854$.

\subsubsection{Number of Contacts}

Eq. 10 shows that the electrical conductivity is inversely proportional to the parameter $\chi$, suggesting linear proportionality between the conductivity $\sigma_{\mu}$ and contacts $m$

$$
\sigma_{\mu} \propto \frac{1}{\chi} \propto 0.15+0.59 m
$$

As reported in section 4.3 we can estimate the number of contacts $m$ through different methods: (i) [29] using Eq. 29, (ii) Stick model and (iii) Cylinder model. The variation of contacts $m$ vs. $\varphi_{C N T}$ is presented in Fig. 8. 


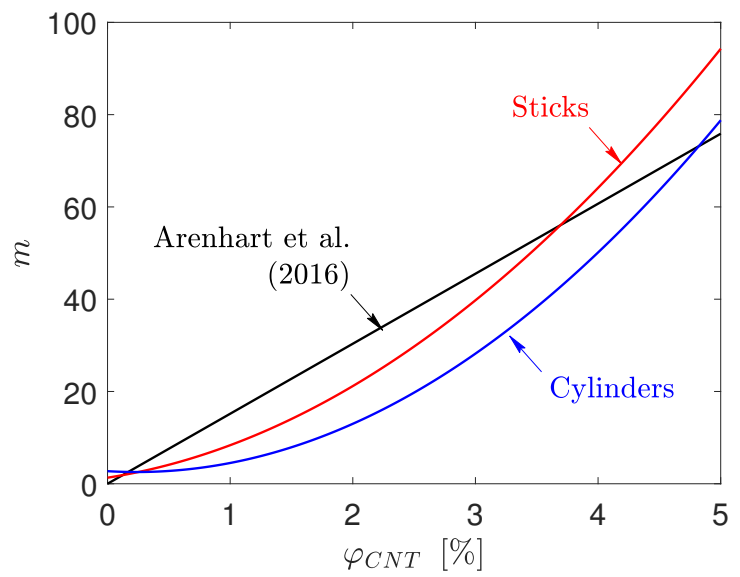

Figure 8: Comparison of number of contacts from three different methods

Eq. 29 suggests that $m$ is linearly proportional to $\varphi_{C N T}$ and $V_{e x}$, hence the straight line in Fig. 8, whereas, both the Stick model and the Cylinder model follow a quadratic equation tendency. The difference between these two models stems from the lack of precision of the Stick model to consider the curvature. Thus, the Stick model overestimates the number of contacts.

\subsubsection{Initial percolation threshold}

The electrical resistivity measured from electrical impedance in section 3 , is analyzed. The variation of electrical resistivity $\rho$ vs. $\varphi_{C N T}$ is presented in Fig. 9. This curve shows that the $\Phi_{P}^{e x p}$ is close to $1.8 \%$. In what follows, this experimental percolation threshold is introduced in the equations.

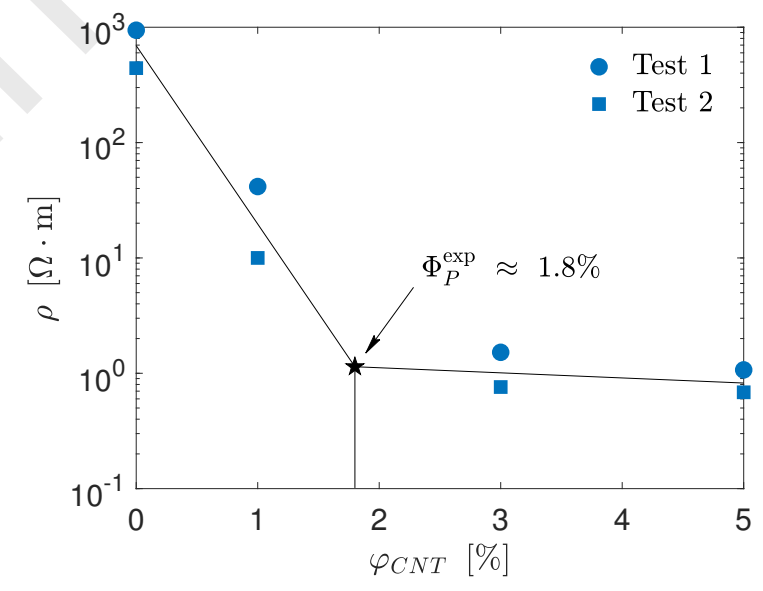

Figure 9: Experimental percolation threshold from [39] 
For $\varphi_{C N T} \leq 1.8 \%$, there is a drastic decrease in resistivity for an increase in CNT volume fraction. This shows the dominant influence of the CNT on the volumic response of the sample, whereas, for volume fraction greater than $1.8 \%$, the resistivity no longer varies. In view of this response, the percolation threshold can be fixed at $1.8 \%$.

As already mentioned, we need the values of $\ell, r, s,\langle\sin (\gamma)\rangle$ and $\Phi_{P}$ to determine the interphase $h$. The value of $\Phi_{P}$ was set to $1.8 \%$ (that measured in the experiments). A summary of the input values to determine $h$ using Eq. 12 is presented in Tab. 1.

Table 1: Input parameters of CNT for $h$ (Eq. 12) and $\sigma_{\mu}$ (Eq. 10)

\begin{tabular}{lcll}
\hline Parameter & symbol & value & units \\
\hline length & $\ell$ & $5 \times 10^{-6}$ & $\mathrm{~m}$ \\
radius & $r$ & $25 \times 10^{-9}$ & $\mathrm{~m}$ \\
waviness & $s$ & 2.4 & - \\
Percolation threshold & $\Phi_{P}^{\exp }$ & 1.8 & $\%$ \\
diameter of contact & $d_{c}$ & $r \times 10^{-6}$ & $\mathrm{~m}$ \\
intrinsic conductivity & $\sigma_{C N T}$ & $1 \times 10^{+6}$ & $\mathrm{~S} \cdot \mathrm{m}^{-1}$ \\
\hline
\end{tabular}

Thanks to the numerical micro-structure, we can estimate $\langle\sin (\gamma)\rangle(\operatorname{section} 4.2)$ and the number of contacts $m$ (using the Cylinder model presented in section 4.3), hence the conductivity. The number of contacts initially is presented in Fig. 10a. In Fig. 10b conductivity is plotted vs. $\varphi_{C N T}$. Recall that the $\varphi_{C N T}$ influences the effective volume fraction as in Eq. 7 and thus the conductivity. 


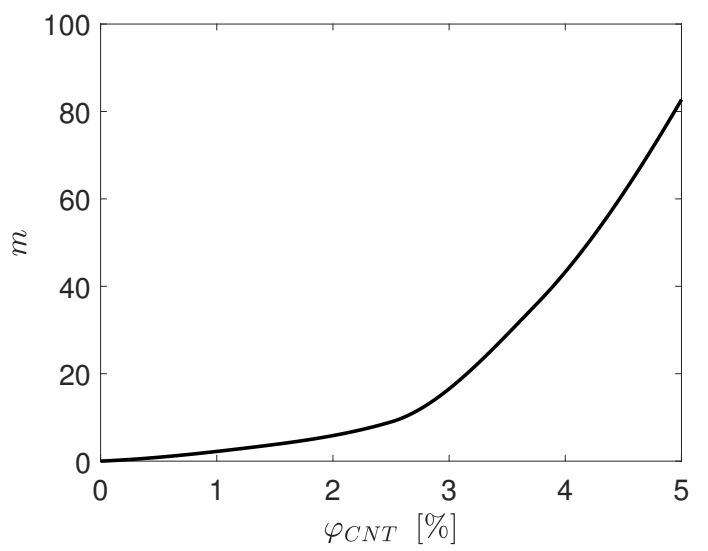

(a) Number of contacts vs. $\varphi_{C N T}$

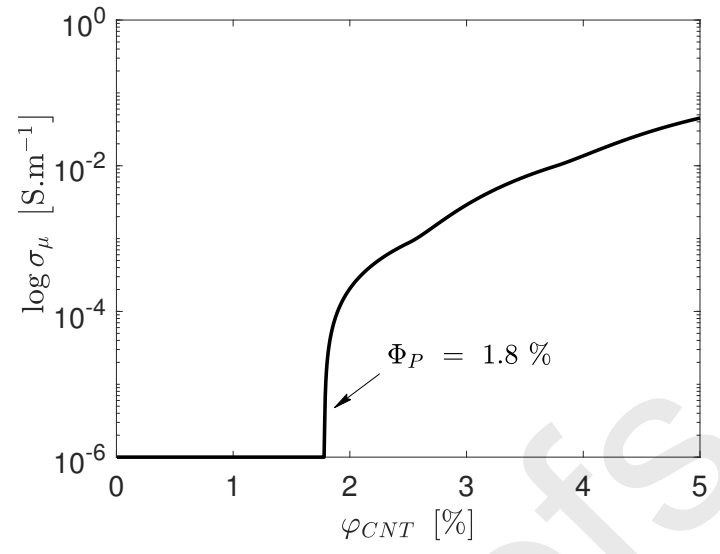

(b) Initial conductivity vs. $\varphi_{C N T}$

Figure 10: Contacts, conductivity and percolation threshold predicted by PEV approach

As the experimental percolation threshold of $1.8 \%$ was used to determine $h$, it is clear that the numerical percolation threshold predicted by the PEV approach is the same as that seen in experiments. The model will estimate a percolation threshold of $1.8 \%$ for any given orientation of CNTs. The only difference would be the values of contacts and conductivity which arise from a different value of $h$. For example, a micro-structure with lower $h$ values will have fewer contacts and thus less conductivity.

\subsection{Piezo-resistive behavior}

Upon application of mechanical loading, the CNTs are allowed to translate and rotate in the polymer matrix. This means that there will be a change in $\langle\sin (\gamma)\rangle$. The variation of $\langle\sin (\gamma)\rangle$ is presented in Fig. 11a. $\langle\sin (\gamma)\rangle$ in Eq. 6 intervenes in the calculation of $\Phi_{P}$. From Eq. 1, we deduce the following proportionality relation between $\Phi_{P}$ and $\langle\sin (\gamma)\rangle$ :

$$
\Phi_{P} \propto \frac{1}{V_{e x}} \propto \frac{1}{\langle\sin (\gamma)\rangle}
$$

thus a variation in $\langle\sin (\gamma)\rangle$ will induce an opposite variation in $\Phi_{P}$. As Fig. 11b demonstrates, $\Phi_{P}$ decreases with increasing strain.

A closer look at Fig. 11a and Fig. 11b shows that the variation occurs at the third digit after the decimal point. These results demonstrate that the variation in conductivity is mainly due to the change in contacts at each stress-state. Therefore, $\Phi_{P}$ is considered to be constant throughout all stress-states. 


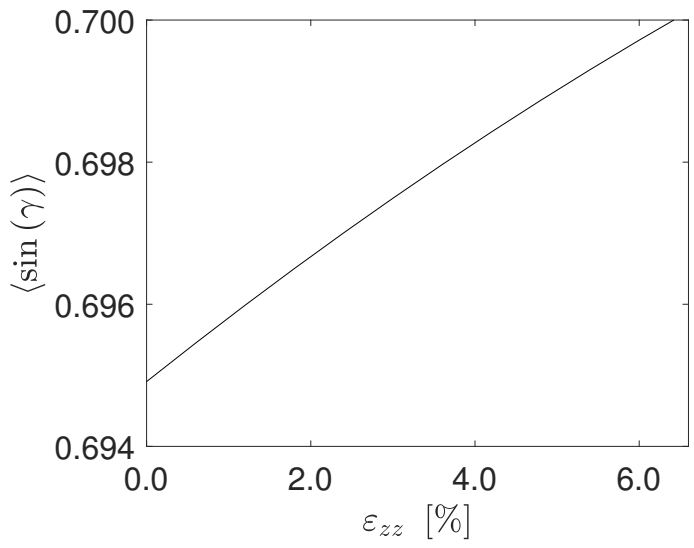

(a) $\langle\sin (\gamma)\rangle$

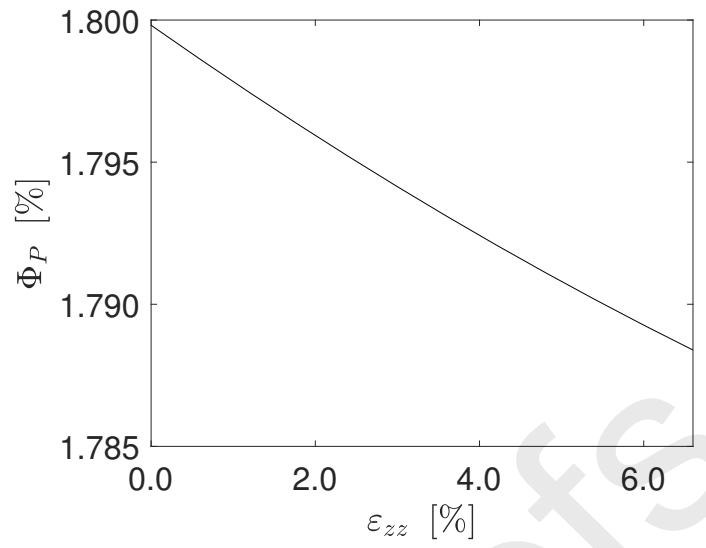

(b) $\Phi_{P}$

Figure 11: Variation of $\langle\sin (\gamma)\rangle$ and $\Phi_{P}$ vs. $\varepsilon_{z z}$

Different micro-structures with different CNT orientations were subjected to mechanical loading and their respective changes in contacts vs. strain were compared. A total of 5 different CNT orientations was tested. Twenty different micro-structures with the same CNT orientation were subjected to mechanical load (in other words, $20 \times 5$ micro-structures). The averages of 20 tests for each test are presented in Fig. 12a. Regardless of the number of drafts drawn, the micro-structures with a CNT orientation other than $0^{\circ}$ do not show monotonic decrease in contacts. 


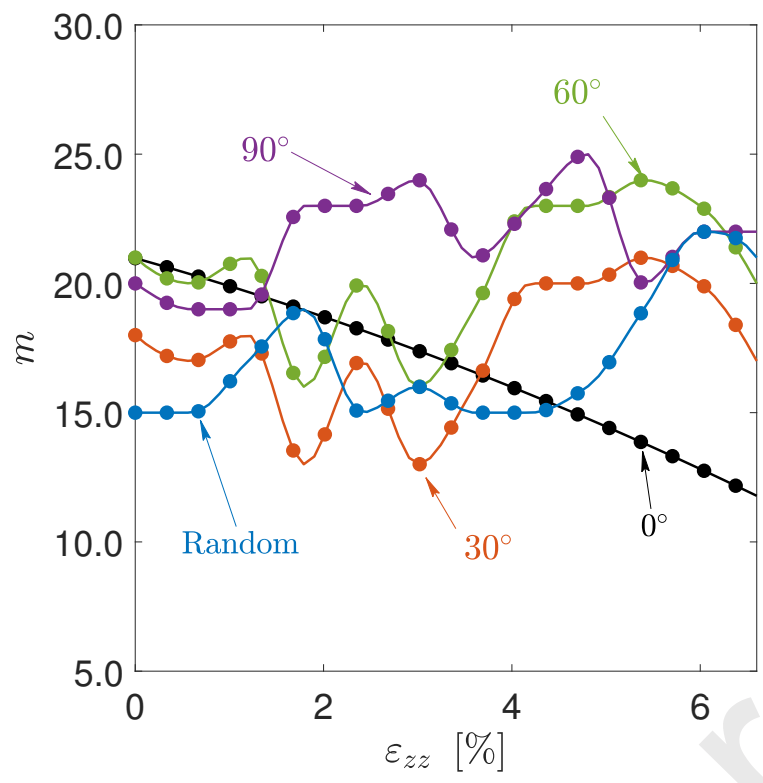

(a) Effect of CNT orientation on $m$ vs. $\varepsilon_{z z}$

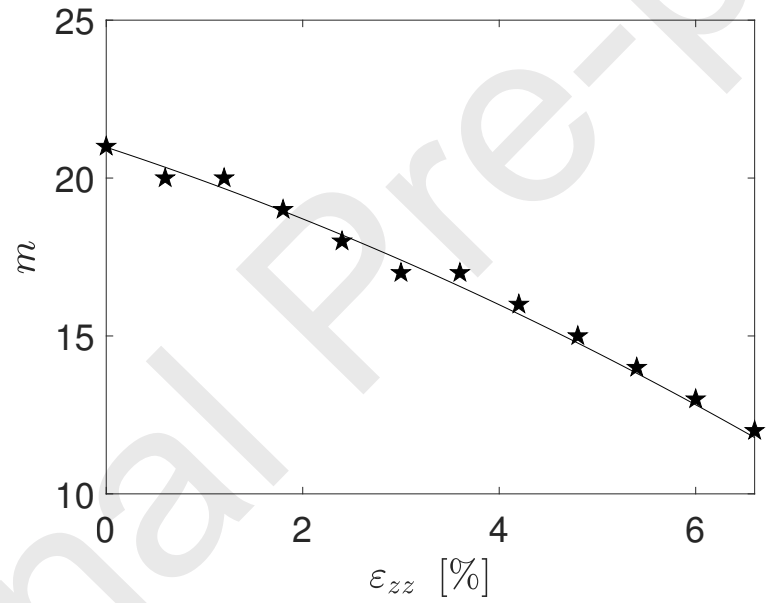

(b) $m$ for $\theta_{\max }=0^{\circ}$ and its $2^{\text {nd }}$ order polynomial fit

Figure 12: Variation of number of contacts with strain for different angular parameters $\theta_{\max }$ (Eq. 26)

Eq. 18 through Eq. 20 together with Eq. 26 and Eq. 27 prove that each draft leads to a slightly different micro-structure even though the angle $\theta_{\max }$ in Eq. 26 is the same. For a given orientation (fixed $\theta_{\max }$ ), the randomness inherent to the generation of micro-structure (section 4.2) gives rise to small oscillations in the data.

The spatial degree of randomness present in the initial micro-structure influences the sites where contacts form or vanish in the successive micro-structure distributions at different stressstates. For this reason, some irregular variations are to be expected even though the strain 
evolves in a monotonic manner (see Fig. 12b).

Having determined the variation in contacts, we can now determine the variation in electrical conductivity (see Fig. 13a) of a micro-structure and thus the variation in resistivity (see Fig. $13 b)$.

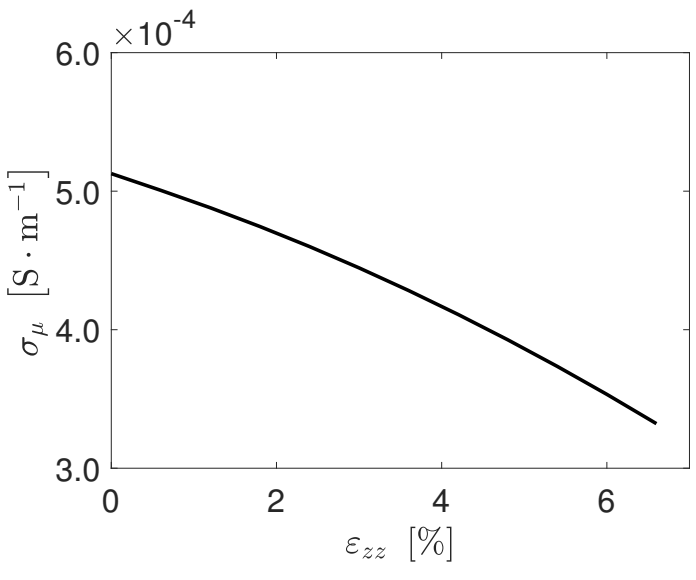

(a) conductivity vs. strain

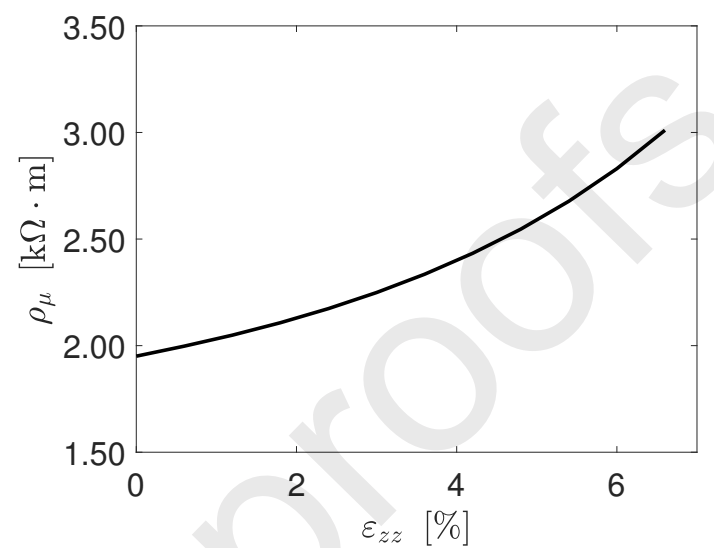

(b) resistivity vs. strain

Figure 13: Evolution of conductivity and resistivity with mechanical strain

In order to determine the variation in resistance over the sample volume at macro-scale, we assume that the macroscopic sample is a homogeneous distribution of a given deformed micro-structure, whose resistance at macro-scale $\left(R_{\varepsilon}\right)$ is:

$$
R_{\varepsilon}=\rho_{\mu} \frac{\ell_{S}}{\pi r_{S}^{2}}
$$

$\ell_{S}$ and $r_{S}$ in Eq. 35 are the length and radius of a cylindrical sample at macro-scale. They were considered to be $10[\mathrm{~mm}]$ and $10[\mathrm{~mm}]$ respectively. Then the variation in resistance between the deformed and initial states is expressed as:

$$
\Delta R=R_{\varepsilon}-R_{0}
$$

The effects of CNT orientation on the response $\Delta R$ were also tested. Fig. 14a, presents the numerical response for different angles with respect to $X$ axis. The micro-structure with $\theta_{\max }=0^{\circ}$ shows a positive and monotonic $\Delta R$ response, whilst other micro-structures with $\theta_{\max }=30^{\circ}, \theta_{\max }=60^{\circ}$ and $\theta_{\max }=90^{\circ}$ show more oscillating responses with reoccurring negative $\Delta R$ values. These tendencies can be clearly seen by the solid lines. As proven by 
Fig. 14a, micro-structures with CNTs orthogonal to the loading directions were retained for comparison. The comparison of a numerical sample using parameters from Tab. 1 and CNTs orthogonal to the loading direction with experimental results is presented in Fig. 14b.

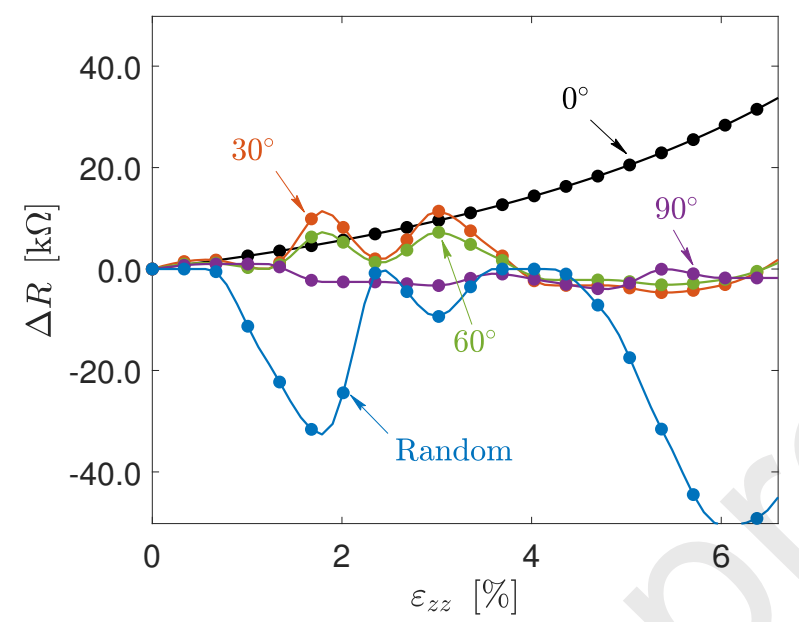

(a) Effect of CNT orientation on $\Delta R$

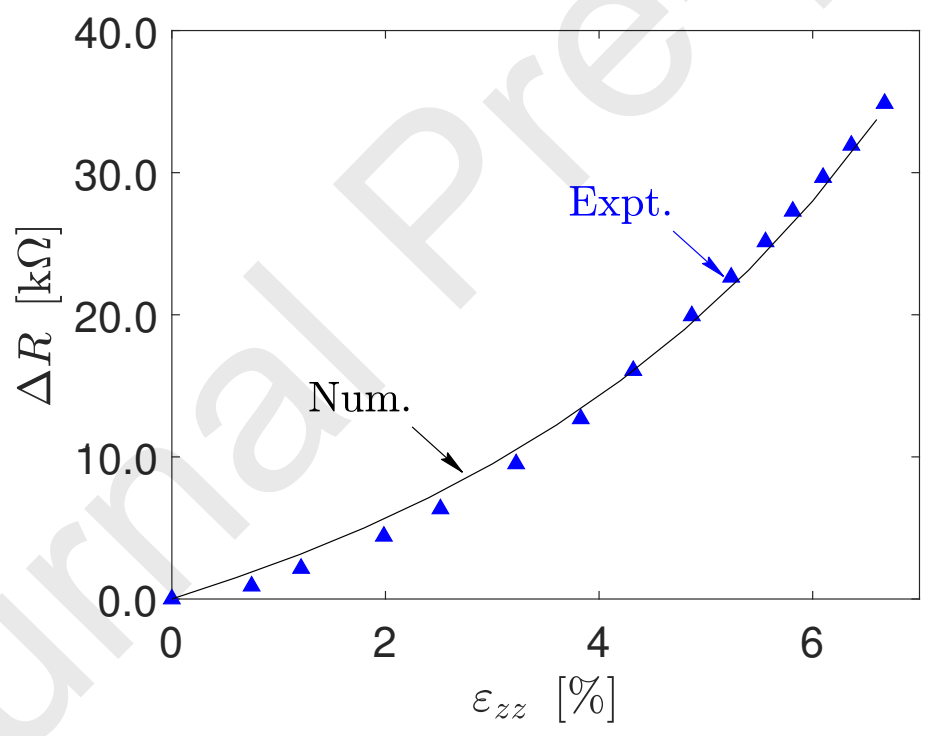

(b) $\Delta R$ for $\theta_{\max }=0^{\circ}$

Figure 14: Variation of $\Delta R$ at the macro-scale with respect to strain and its comparison with experimental results

\section{Conclusion}

A model based on the Percolation Excluded Volume (PEV) approach supplemented by Finite Element simulation and experimental data is proposed to predict the piezo-resistive 
behavior of EPDM-CNT composites.

In this model, the interphase thickness is not only dependent on CNT geometry (length, radius, waviness) but also on the $\mathrm{CNT}$ mean spatial orientation. The interphase thickness increases as the CNTs change from orthogonally oriented to parallel orientation with the loading direction. When the mechanical loading is applied, it is found that the variation in the mean orientation does not induce significant variation of the percolation threshold, which was therefore assumed constant in the simulation. In the proposed model, the number of tunneling contacts was found to play a key role. This number of contacts was calculated through the finite element model.

A realistic stress-strain law (derived from quasi-static experiments on EPDM-CB-CNT composite samples) was assigned to a numerical sample. The influence of the electrical field on the mechanical fields is not formulated in the present model. In the experimental study, the stress-strain relationship and the electrical resistance were measured simultaneously during the compressive tests and they did not present non-regular (or abrupt) evolutions (stiffening or softening) as compared to the compression of a PNC sample free of voltage. These stress-strain curves were used in the FE model. Therefore, in a certain way, the electromechanical effects are indirectly taken into account.

Since the CNTs were modelled as fibers in continuous media, the effective properties were employed (i.e. the effective volume fraction) of an equivalent fiber using an analytical model. However, to model the materials accurately, the equivalent fiber including the CNT and the surrounding interphase should be considered. This will be performed in future work.

The displacement fields calculated during the compression-test were used to evaluate the CNT displacements. The main limitation inherent to the methodology used might be that the mechanical coupling between CNT and the EPDM matrix is not resolved. Instead, a weak coupling is used to infer the CNT displacements by the classical interpolation procedure applied in finite element methods. The finite element simulation associated with this weak coupling hypothesis contribute to providing CNT displacements which come closer to the real one, although they still need to be improved.

However, this simplified hypothesis enables the model to confirm the ability and efficiency of the PEV approach to predict the experimental results at low simulation costs. 


\section{Acknowledgments}

The authors would like to acknowledge "la Région Centre Val de Loire" for funding this work via the CLEBER project.

\section{Declaration of Interest}

The author(s) declare no potential conflicts of interest with respect to the research, authorship, and/or publication of this article.

\section{References}

[1] S. C. Baxter, C. T. Robinson, Pseudo-percolation: Critical volume fractions and mechanical percolation in polymer nanocomposites, Composites Science and Technology 71 (10) (2011) 1273-1279. doi:10.1016/j.compscitech.2011.04.010.

[2] Y. Zhang, Y.-J. Heo, M. Park, S.-J. Park, Recent advances in organic thermoelectric materials: Principle mechanisms and emerging carbon-based green energy materials, Polymers 11 (1). doi:10.3390/polym11010167.

URL https://www.mdpi.com/2073-4360/11/1/167

[3] S. Riaz, S.-J. Park, Thermal and mechanical interfacial behaviors of graphene oxidereinforced epoxy composites cured by thermal latent catalyst, Materials 12 (8). doi: $10.3390 / \mathrm{ma1} 2081354$.

URL https://www .mdpi.com/1996-1944/12/8/1354

[4] Y. Zhang, S.-J. Park, Effective reinforcement of melamine-functionalized ws2 nanosheets in epoxy nanocomposites at low loading via enhanced interfacial interaction, Macromolecular Research 28 (2020) 1116-1126. doi:https://doi.org/10.1007/s13233-020-8151-8.

[5] Y. Zhang, J. R. Choi, S.-J. Park, Enhancing the heat and load transfer efficiency by optimizing the interface of hexagonal boron nitride/elastomer nanocomposites for thermal management applications, Polymer 143 (2018) 1-9. doi:https://doi.org/10.1016/j. polymer. 2018.03.067.

URL https://www.sciencedirect.com/science/article/pii/S0032386118302878 
[6] N. Vu-Bac, R. Rafiee, X. Zhuang, T. Lahmer, T. Rabczuk, Uncertainty quantification for multiscale modeling of polymer nanocomposites with correlated parameters, Composites Part B: Engineering 68 (2015) 446-464. doi : https : //doi .org/10.1016/j . compositesb. 2014.09 .008$.

URL https://www.sciencedirect.com/science/article/pii/S1359836814004107

[7] V. Sankar, K. Balasubramaniam, R. Sundara, Insights into the effect of polymer functionalization of multiwalled carbon nanotubes in the design of flexible strain sensor, Sensors and Actuators A: Physical (2021) 112605doi:https://doi.org/10.1016/j.sna. 2021. 112605.

URL https://www.sciencedirect.com/science/article/pii/S0924424721000662

[8] Y. Sun, H.-D. Bao, Z.-X. Guo, J. Yu, Modeling of the Electrical Percolation of Mixed Carbon Fillers in Polymer-Based Composites, Macromolecules 42 (1) (2009) 459-463. doi: 10.1021/ma8023188.

[9] J. Njuguna, F. Ansari, S. Sachse, H. Zhu, V. M. Rodriguez, 1 - Nanomaterials, nanofillers, and nanocomposites: types and properties, in: J. Njuguna, K. Pielichowski, H. Zhu (Eds.), Health and Environmental Safety of Nanomaterials, Woodhead Publishing, 2014, pp. 3 27. doi:10.1533/9780857096678.1.3.

[10] M. L. Clingerman, E. H. Weber, J. A. King, K. H. Schulz, Synergistic effect of carbon fillers in electrically conductive nylon 6,6 and polycarbonate based resins, Polymer Composites 23 (5) (2002) 911-924. doi:10.1002/pc.10488.

[11] W. Thongruang, R. J. Spontak, C. M. Balik, Correlated electrical conductivity and mechanical property analysis of high-density polyethylene filled with graphite and carbon fiber, Polymer 43 (8) (2002) 2279-2286. doi:10.1016/S0032-3861(02)00043-5.

[12] Y. Chen, S. Wang, F. Pan, J. Zhang, A Numerical Study on Electrical Percolation of Polymer-Matrix Composites with Hybrid Fillers of Carbon Nanotubes and Carbon Black, Journal of Nanomaterials 2014 (2014) e614797. 
[13] W. Mark, K. Musa.R, Estimation of the volume resistivity of electrically conductive composites, Journal of Composites 18 (6) (1997) 711-725. doi:doi:10.1002/pc. 10324.

[14] I. Balberg, C. H. Anderson, S. Alexander, N. Wagner, Excluded volume and its relation to the onset of percolation, Physical Review B 30 (7) (1984) 3933-3943. doi:10.1103/ PhysRevB.30.3933.

[15] L. Onsager, The Effects of Shape on the Interaction of Colloidal Particles, Annals of the New York Academy of Sciences 51 (4) (1949) 627-659. doi:10.1111/j.1749-6632.1949. tb27296.x.

[16] L. Berhan, A. M. Sastry, Modeling percolation in high-aspect-ratio fiber systems. I. Softcore versus hard-core models, Physical Review E 75 (4) (2007) 041120. doi:10.1103/ PhysRevE.75.041120.

[17] N. Ibarra-Avalos, A. Gil-Villegas, A. Martinez Richa, Excluded volume of hard cylinders of variable aspect ratio, Molecular Simulation 33 (6) (2007) 505-515. doi : 10.1080/ 08927020701191349.

[18] H. Scher, R. Zallen, Critical Density in Percolation Process, The Journal of Chemical Physics 53 (9) (1970) 3759-3761. doi:10.1063/1.1674565.

[19] I. Balberg, N. Binenbaum, N. Wagner, Percolation Thresholds in the Three-Dimensional Sticks System, Physical Review Letters 52 (17) (1984) 1465-1468. doi:10.1103/ PhysRevLett.52.1465.

[20] G. E. Pike, C. H. Seager, Percolation and conductivity: A computer study. I, Physical Review B 10 (4) (1974) 1421-1434. doi:10.1103/PhysRevB.10.1421.

[21] I. Balberg, N. Binenbaum, Computer study of the percolation threshold in a twodimensional anisotropic system of conducting sticks, Physical Review B 28 (7) (1983) 3799-3812. doi:10.1103/PhysRevB.28.3799.

[22] S. F. Wang, A. A. Ogale, Continuum space simulation and experimental characterization of electrical percolation behavior of particulate composites, Composites Science and Technology 46 (2) (1993) 93-103. doi:10.1016/0266-3538(93)90165-D. 
[23] M. Taya, W. J. Kim, K. Ono, Piezoresistivity of a short fiberrelastomer matrix composite, Mechanics of Materials 28 (1998) 53-59.

[24] Y. Zare, K. Y. Rhee, Development of a conventional model to predict the electrical conductivity of polymer/carbon nanotubes nanocomposites by interphase, waviness and contact effects, Composites Part A: Applied Science and Manufacturing 100 (2017) 305-312. doi:10.1016/j.compositesa.2017.05.031.

[25] S. Gong, Z. H. Zhu, J. Li, S. A. Meguid, Modeling and characterization of carbon nanotube agglomeration effect on electrical conductivity of carbon nanotube polymer composites, Journal of Applied Physics 116 (19) (2014) 194306, publisher: American Institute of Physics. doi:10.1063/1.4902175.

[26] Y. Zare, K. Y. Rhee, Calculation of tunneling distance in carbon nanotubes nanocomposites: effect of carbon nanotube properties, interphase and networks, Journal of Materials Science 55 (13) (2020) 5471-5480. doi:10.1007/s10853-019-04176-2.

[27] T. Takeda, Y. Shindo, Y. Kuronuma, F. Narita, Modeling and characterization of the electrical conductivity of carbon nanotube-based polymer composites, Polymer 52 (17) (2011) 3852-3856. doi:10.1016/j . polymer. 2011.06.046.

[28] X. Zeng, X. Xu, P. M. Shenai, E. Kovalev, C. Baudot, N. Mathews, Y. Zhao, Characteristics of the Electrical Percolation in Carbon Nanotubes/Polymer Nanocomposites, The Journal of Physical Chemistry C 115 (44) (2011) 21685-21690, publisher: American Chemical Society. doi:10.1021/jp207388n.

[29] R. G. Arenhart, G. M. O. Barra, C. P. Fernandes, Simulation of percolation threshold and electrical conductivity in composites filled with conductive particles: Effect of polydisperse particle size distribution, Polymer Composites 37 (1) (2016) 61-69. doi:10.1002/pc. 23155.

[30] R. Razavi, Y. Zare, K. Y. Rhee, A two-step model for the tunneling conductivity of polymer carbon nanotube nanocomposites assuming the conduction of interphase regions, 
RSC Advances 7 (79) (2017) 50225-50233, publisher: The Royal Society of Chemistry. doi:10.1039/C7RA08214B.

[31] Z. Liu, W. Peng, Y. Zare, D. Hui, K. Y. Rhee, Predicting the electrical conductivity in polymer carbon nanotube nanocomposites based on the volume fractions and resistances of the nanoparticle, interphase, and tunneling regions in conductive networks, RSC Advances 8 (34) (2018) 19001-19010, publisher: The Royal Society of Chemistry. doi:10.1039/ C8RA00811F.

[32] M. Haghgoo, M. K. Hassanzadeh-Aghdam, R. Ansari, A comprehensive evaluation of piezoresistive response and percolation behavior of multiscale polymer-based nanocomposites, Composites Part A: Applied Science and Manufacturing 130 (2020) 105735. doi:10.1016/j.compositesa.2019.105735.

[33] A. Ghazavizadeh, M. Baniassadi, M. Safdari, A. A. Atai, S. Ahzi, S. A. Patlazhan, J. Gracio, D. Ruch, Evaluating the Effect of Mechanical Loading on the Electrical Percolation Threshold of Carbon Nanotube Reinforced Polymers: A 3D Monte-Carlo Study, Journal of Computational and Theoretical Nanoscience 8 (10) (2011) 2087-2099. doi:10.1166/jctn.2011.1930.

[34] B. Hu, N. Hu, Y. Li, K. Akagi, W. Yuan, T. Watanabe, Y. Cai, Multi-scale numerical simulations on piezoresistivity of CNT/polymer nanocomposites, Nanoscale Research Letters 7 (1) (2012) 402. doi:10.1186/1556-276X-7-402.

[35] T. Tallman, K. W. Wang, An arbitrary strains carbon nanotube composite piezoresistivity model for finite element integration, Applied Physics Letters 102 (1) (2013) 011909. doi: $10.1063 / 1.4774294$.

[36] A. S. Tsiamaki, G. I. Giannopoulos, S. K. Georgantzinos, N. K. Anifantis, 12 - Finite Element Modeling of Nanotubes, in: R. Rafiee (Ed.), Carbon Nanotube-Reinforced Polymers, Micro and Nano Technologies, Elsevier, 2018, pp. 291-310. doi:10.1016/ B978-0-323-48221-9.00012-1.

[37] A. K. Manta, K. I. Tserpes, 13 - Multiscale Simulation of Impact Response of Carbon 
Nanotube/Polymer Nanocomposites, in: R. Rafiee (Ed.), Carbon Nanotube-Reinforced Polymers, Micro and Nano Technologies, Elsevier, 2018, pp. 311-345. doi:10.1016/ B978-0-323-48221-9.00013-3.

[38] N. Vu-Bac, T. Rabczuk, X. Zhuang, 15 - Continuum/Finite Element Modeling of Carbon Nanotube-Reinforced Polymers, in: R. Rafiee (Ed.), Carbon Nanotube-Reinforced Polymers, Micro and Nano Technologies, Elsevier, 2018, pp. 385-409. doi:10.1016/ B978-0-323-48221-9.00015-7.

[39] N. Penvern, A. Langlet, M. Gratton, M. Mansion, N. Aït Hocine, Experimental characterization of the quasi-static and dynamic piezoresistive behavior of multi-walled carbon nanotubes/elastomer composites, Journal of Reinforced Plastics and Composites 39 (7-8) (2020) 299-310. doi:10.1177/0731684420901754.

[40] W. S. Bao, S. A. Meguid, Z. H. Zhu, M. J. Meguid, Modeling electrical conductivities of nanocomposites with aligned carbon nanotubes, Nanotechnology 22 (48) (2011) 485704, publisher: IOP Publishing. doi:10.1088/0957-4484/22/48/485704.

[41] W. Fang, H. W. Jang, S. N. Leung, Evaluation and modelling of electrically conductive polymer nanocomposites with carbon nanotube networks, Composites Part B: Engineering 83 (2015) 184-193. doi:10.1016/j.compositesb.2015.08.047.

[42] B. Loung, Distance bewteen 2 cylinders in 3D (2020).

URL https://fr.mathworks.com/matlabcentral/fileexchange/ 68796-distance-bewteen-2-cylinders-in-3d 